\title{
Rubber Price Effect on Exchange Rate: A Bayesian Mixture Model Approach
}

\author{
${ }^{*}$ Seuk Yen Phoong, Mohd Tahir Ismail \\ University Science Malaysia, Penang, Malaysia \\ *yen_phoong@hotmail.com
}

\begin{abstract}
Mixture model is a probabilistic model that denotes the presence of subpopulations within an overall population meanwhile finite mixture model is a mixture model with finite-dimensional. In this paper, finite mixture model is applied and the application of Bayesian method to fit finite mixture model is popular and these application is adopt in the present study in order to explore the relationship between rubber price and exchange rate for Malaysia, Thailand, Philippines and Indonesia. Exchange rate plays leading role for a country because it represents the development for that country. The changes of exchange rate influence the flows of investment either the import or export prices for a nation's. While another data set that adopt is rubber price. Rubber is an economic commodity that prospers in tropical climate. It is an important raw material because the latex that extracted is the primary source of natural rubber that enables to produce many useful products such as tires, surgical gloves, industrial hoses, rubber sheeting, industrial form parts and others household rubber products. Results found that rubber price effect on the change of exchange rate for Malaysia, Thailand, Philippines and Indonesia.
\end{abstract}

Keywords: Finite mixture model, Bayesian method, rubber price and exchange rate

\section{Introduction}

Finite mixture model is a mixture of other distributions in modeling a statistical distribution. This model also provides a natural representation of heterogeneity in a finite number of latent classes. In addition, finite mixture model also known as latent class models or unsupervised learning models. A mixture of $k$ components in finite mixture model has $N$ random variables and each component belonging to the same parametric family of distributions but with different parameters. Furthermore, Lindsay (1989) reviewed that finite mixture model is a semiparametric estimator of the density. In statistics, finite mixture model is increasingly getting attention over the years because of its flexibility in both practical and theoretical point of view. The usefulness of applied finite mixture model include modeling of over-dispersed data, fitting the zero-inflated or hurdle models, estimating heavy-tailed densities, modeling heterogeneity in a cluster analysis, calculating of switching regressions, allows measuring of mixture of univariate distribution and in estimating a mixing probability in the data. In addition, finite mixture model also able to measure the observable variables, describe the complex system on the data analysis and measure the sensitivity and specificity of the diagnostic and screening procedures. Bayesian method is one of the statistical tools that adopted to fit the finite mixture model and successfully applied in many fields such as medical, statistics, reliability, jurisprudence, financial, economic, social sciences, meteorology, hydrology and mechanics. Bayesian method is developed from the Bayes' theorem and it is a general approach in calibrate and identify the models. Moreover, Bayesian method also used to estimate parameters of the model and makes predictions for new observations. There are three concepts that Bayesian method built on which are prior distribution, likelihood function and posterior distribution. Prior distribution is the information of the model parameters before using the observed data. While likelihood function demonstrates the information that involved in the observed data. The posterior distribution is well known as the weighted average between prior distribution and likelihood function. Therefore, the prior distribution and likelihood function in Bayesian method acts as antecedents and the results is then derives in the posterior distribution which based on the two antecedents given.

Bayesian method often shows consistency characteristic which means that the parameter estimates are close to the predictive distributions. Furthermore, Bayesian method has asymptotic properties which provided remarkable result. The advantage of adopted Bayesian method is obtains a more accurate prediction with proper prior distributions. This method allow to test the hypotheses without predetermining the outcome of the test according to the selection of the sample size, and also need not to pre-specify an arbitrary level of significance. In this study, a two-component mixture of normal distribution is measure by using Bayesian method in which to explore the relationship between rubber price and exchange rate. Rubber is a raw material that prospers in tropical climate. The scientific name 
for rubber tree is known as Hevea brasiliensis in which mostly growth in Malaysia, Thailand, Indonesia, Philippines, Vietnam, China and India in Asia, as well as in Nigeria, Liberia and Gabon in Africa. Furthermore, rubber is an importance commodity because the latex that extracted from the tree is the primary source of natural rubber. Latex is a milky white sap that has elastic property. In addition, latex is a renewable resource that can be sustainable tapped without harming the tree. Rubber with useful properties such as highly elastic, water resistant and nonconductive characterizes, enables it became one of the popular raw material. The latex is then collected for processing and forms dry rubber or latex concentrate. Thus, the use of rubber is widespread, ranging from household to industrial products. The rubber products are erasers, belting, footwear, hoses, surgical goods, rubberized cloth, tires, tubes, door and window profiles, gloves and toy balloons. Besides that, rubber also used to produce aircraft tires and inner tubes.

Another data set that adopt in the present study is exchange rate. In financial, exchange rate regarded as the value of currency for one country in terms of another currency. A currency become more valuable as the demand for it is greater than the available supply. Moreover, the demand of currency can be affected by inflation rate, interest rate, trade balance, political stability, internal harmony and regulation from government. Exchange rate is important for one country because it facilitating international trade of goods between countries and allows conversion of one country's currency into another. Furthermore, exchange rate is increases with the strengthened or increased of currency's value. The data set that applied for the present study is financial returns data. In a real financial returns data, there are reasonable to have mixture model or a jump-diffusion model since financial returns always exhibit different properties during crisis times and normal situations passing the time. Thus, Bayesian method is an effective tool in fitting finite mixture model. While the distribution that applied in this study is a twocomponent mixture of normal distributions. The rest of the sections that included in this paper are as follow. Section 2 proposes the literature review for Bayesian method. Section 3 presents the sample and data that adopted in this paper. Section 4 describes the methodology and section 5 is the results and discussions. Lastly, section 6 presents the conclusion of the paper.

\section{Literature Review}

This section summarized the reviews from journal, articles and books that related to Bayesian method. Bayesian method is first proposed by Laplace (1986). This method is then widely applied to estimate the parameters of finite mixture model. The application of Bayesian method to fit finite mixture model was drawn great response. Moreover, Bayesian method is suitable in time series analysis because it involves highly nonlinear dynamic structure which will causes difficulties in prior specification and in posterior evaluation. Schervish and Tsay (1988) applied Bayesian approach of autoregressive time series modeling to handle the outliers and missing observations within the model. Furthermore, Abraham and Box (1979) also adopted Bayesian formulation to detect the outliers in a univariate model. According to Liu and Tiao (1980), Bayesian method is applied in order to handle the random coefficient autoregressive models. Meanwhile Cooper and Herskovits (1992) studied the Bayesian method in constructing the probabilistic networks from data. In addition, Cooper and Herskovits (1992) also reviewed Bayesian method to address the missing data, latent variables problems and also studied the pharmaco economic data. Moreover, Jones (1998) reviewed the Bayesian method to examine the high frequency augmentation (HFA) which is a simple method that enables to apply in univariate or multivariate diffusion and jumpdiffusion process. Then, the Markov Chain Monte Carlo based on Bayesian method is proposed to attain the posterior distributions of the parameters and examined the missing or latent data. The HFA is then found that robust to a variety of common features in financial and economic data.

Furthermore, Doss and Huffer (2003) studied a mixture of Dirichlet process prior on survival distribution by two methods, including Bayesian method via Markov chain Monte Carlo and sequential important sampling. Result depicts that sequential important sampling is a method that well suited to address missing data problems than Bayesian method in this case. While the survival data that applied is the breast cancer data. Doss and Huffer (2003) and Kottas (2006) have similarity in analyzing survival distribution using Bayesian method. Kottas (2006) studied the Bayesian method in which based on the mixture of Weibull distribution for kernel and the Dirichlet process prior for the mixing distribution. The analysis is then used to model the survival populations. Berry (2004) reviewed Bayesian method to examine medical field in order to improve the efficiency of adaptive trials. Bayesian method is found that effective in estimating clinical trials. Properties such as minimizing costs, length of trial and number of patients treated are obtained from the analysis. Besides that, maximizing the information about slope 
dose-response curve and effective treatment of patients in the trial also found when Bayesian method is used in the analysis. In addition, Cano et al. (2004) applied Bayesian networks as data mining technique to examine meteorology data. The observed data that being analyzed include two variables which are daily rainfall and maximum wind speed for a network of 100 stations in Iberian Peninsula.

In addition, Martina et al. (2008) also applied Bayesian method to define rainfall thresholds for flood warning system in Sieve River, Italy. Martina et al. (2008) described that the Bayesian method is suitable to predict flood warning because it is easier to be understood and applied by non technical users. Besides that, Madigan et al. (2010) applied Bayesian method to examine the drug safety problems and this method is found that useful in analysis the pharmaceutical and health distributions. The main reason is that Bayesian method has high dimensionality of the data. Bayesian method also implemented in order to analysis the cost-effectiveness problems by Armero et al. (2010). Cost-effectiveness analysis is a methodology that accounts two or more alternative treatments which include the costs and consequences for each treatment. In finance, Bayesian method is widely used to concern the short-term interest rate. Feng and Xie (2012) studied the Bayesian method to measure the parameters of the historical data of United States Treasury bills. The Bayesian method is proposed to analysis the short-term interest rate follows the Cox-Ingersoll-Roll (CIR) model. Then, the Markov Chain Monte Carlo based on Gibbs sampler algorithm is applied in order to construct latent data for the Treasury bills yields. According to Cipriani et al. (2012), Bayesian method is adopted to study the data of an experimental financial market. Five types of trading decisions such as private-information based, noise, herd, contrarian and irresolute are measured via Bayesian method and the model that best fit the data is investigated. Result show that irresolute trading decision is the best model that fitted the data. The study of Jacquier and Polson (2012) has similarity with Cipriani et al. (2012) in adopted Bayesian method to model the finance data. Jacquier and Polson (2012) applied Bayesian method in determined the input needed for the optimal asset allocation in finance and the asset allocation that mentioned are the expected excess return and volatility of the risky asset.

Sample and Data: The data that adopted in this paper are rubber price and returns nominal change of exchange rate per month for Malaysia, Thailand, Philippines and Indonesia. The data is obtained from July 2005 until September 2012. The data for exchange rate is collected from yahoo finance meanwhile the data for rubber prices is obtained from index mundi. The statistical software that applied in this paper is SAS version 9.3.

\section{Methodology}

In this study, the important step before data analysis is identifying the number of components $k$. In finite mixture models, number of components also known as a priori and is not a parameter to be estimated. Identifying number of components before the analysis of data is an important step in which to avoid invalidity of the result. There are two common used statistical methods which aim to estimate number of components that are Akaike Information Criterion (AIC) and Bayesian Information Criterion (BIC). Akaike Information Criterion and Bayesian Information Criterion are both penalized likelihood criteria that usually apply to find the best predictor subsets in regression. Therefore, both AIC and BIC are applied in this study because it is a criterion for model selection among a finite set of models. Akaike Information Criterion (AIC) is a measure of goodness of fit for a statistical model. This model selection was published by Akaike (1974). In general case, the formula to compute AIC is

AIC $=-2 \operatorname{In}(g)+2 h$

Where $g$ denotes the maximized value of likelihood function for the estimated model and $h$ denotes the number of parameters in the model. The model with the smallest AIC value indicates that preferable model in compared with others. In addition, AIC is asymptotically effective and unbiased for the sample sizes larger than 30 .

Moreover, Bayesian Information Criterion was proposed by Schwarz (1978). This criterion is an estimate of a function of the posterior probability of a model and the lower BIC denotes that a model is considered as a true model. The BIC is an asymptotic result which derived under the assumptions that the data distribution is in the exponential family. The formula for BIC is

$\mathrm{BIC}=-2 \operatorname{In}(H)+g \operatorname{In}(n)$

Where $H$ denotes the maximized value of the likelihood function for the estimated model

$g$ denotes the number of free parameters to be estimated

$n$ denotes the number of observation 
However, if the model errors or disturbances are normally distributed under the assumption, the equation above would changed to

$$
B I C=n \ln \left(\frac{R S S}{n}\right)+g \ln (n)
$$

where RSS represented the residual sum of squares from the estimated model. In addition, the BIC found that more strongly in penalized the free parameters than AIC.

In this study, both AIC and BIC are adopted in order to determine the number of components $k$. After that, Bayesian method is applied to fit a $k$-component mixture of normal distribution. In this section, the formula for three concepts of Bayesian method include prior density, likelihood function and posterior distribution are propose. These three concepts is importance in describing the results. While the general formula for Bayesian mixture model is denotes as

$$
P\left(x_{i} \mid \psi\right)=\sum_{j=1}^{j} n_{j} P\left(x_{i} \mid \psi_{j}\right)
$$

where $P(x \mid \psi)$ represents the probability density with observations $x_{1}, \ldots, x_{n}$ and the number of components is known. Moreover, $n_{j}$ demonstrate the $j$-th sample $n$. The purpose of adopted Bayesian inferences is to learn the parameters that characterize the data generating process given observed data. In this statistical inference, parameter vector $\theta$ is considered as random vector whose probability distribution describes the true value of $\theta$. Prior to analyzing the knowledge of data $x$ about $\theta$ which summarized by the probability density function and this density is then known as prior density.

The likelihood function denotes the summarized of all the information about the model parameters that available in the data. In this concept, the relationship between the observed and predicted values are assumed as the errors follow with a normal distribution and it can be denotes as

$$
Y=Q+\varepsilon
$$

where error, $\varepsilon=N\left(0, \sigma^{2}\right)$ and $Q$ is the function of the observed input data $I$. This equation also can know as

$$
Y=\mathrm{f}(\mathrm{I}, \theta)+\varepsilon
$$

when $\theta$ represent as the model parameters. With the $n$ observation of the likelihood function, identically independent distribution is given by the product of individual probability distribution as

$$
H(\theta)=\frac{1}{(\sqrt{2 \pi})^{n} \cdot \sigma^{n}} \exp \left(-\frac{1}{2 \sigma^{2}} \sum_{i=1}^{n}\left(Y-Q_{i}\right)^{2}\right)
$$

where $\sigma^{2}$ represents the variance of the residuals.

The posterior distribution $P(\theta \mid x)$ is the update information of the prior distribution $P(\theta)$ with the likelihood function $P(x \mid \theta)$. Bayesian method can be defined as

where $P$ represents probability density

$$
P(\theta \mid x)=\frac{P(\theta) P(x \mid \theta)}{P(x)}
$$

$\theta$ represents the parameters in prior distribution

$x$ represents the set of data

$P(\theta)$ denotes the prior distribution of the possible $\theta$ values

$P(x \mid \theta)$ denotes the likelihood function of the probabilistic data

$P(x)$ is a probability of the evidence which is constant

$P(\theta \mid x)$ is the posterior distribution of $\theta$ given the observed data $x$

From the prior observed data, the probabilistic pattern could be in known or unknown form to find the probability in prior determination. The denominator of the posterior distribution contains prior predictive distribution as the following

$$
P(x)=\int P(x \mid \theta) P(\theta) d \theta
$$

The posterior distribution $P(\theta \mid x)$ interprets the likelihood function into a proper probability distribution over the unknown parameters. This can be summarized as any probability distribution by computing the quantiles, means, standard deviation, expected values and others.

\section{Results and Discussion}

The determination of number of components $k$ in finite mixture model is an important step in order to avoid invalidity of the results. This is because choosing the improper number of components may lead to 
different results. In this study, model selection criterion such as Akaike Information Criterion (AIC) and Bayesian Information Criterion (BIC) are adopted to fit the increasing number of components. Then, the most suitable number of component can be concluded by referring to the smallest value of the model selection criteria. Table 1 displays that the number of parameters, value of log-likelihood, AIC and BIC for the increasing number of components. According to table 1, LL denotes the value of log-likelihood in which evaluated at the posterior means of the parameters that collected from the iteration history. While the AIC that listed in table 1 represents the Akaike Information Criterion and the formula to calculate AIC is define as AIC $=-2 \mathrm{LL}+2 p$, where $p$ denotes the number of parameters in the model. Meanwhile BIC denotes the Bayesian Information Criterion and define as BIC $=-2 \mathrm{LL}+p \operatorname{In}(n)$, where $p$ represent the number of parameters and $n$ denotes the number of observations.

Table 1: Information based Model Selection Criteria

\begin{tabular}{lllll}
\hline Number of component & $\begin{array}{l}\text { Number of } \\
\text { parameter }\end{array}$ & LL & AIC & BIC \\
\hline$k=2$ & 7 & 932.5326 & -1851.1 & -1824.2 \\
$k=3$ & 11 & 922.2665 & -1822.5 & -1780.3 \\
$k=4$ & 15 & 927.6650 & -1825.3 & -1767.7 \\
$k=5$ & 19 & 930.8983 & -1823.8 & -1750.8 \\
$k=6$ & 23 & 932.8252 & -1819.7 & -1731.3 \\
\hline
\end{tabular}

The number of component with the smallest value of AIC or BIC denotes that better the fits. Therefore, the AIC and BIC values for $k=2$ are found that smaller value than others component and this indicates that $k=2$ is more appropriate in used in this study. Due to the result that obtained from the previous step, Bayesian method is then fitted by a two-component mixture of normal distributions in modeling the relationship between the rubber price and exchange rate. This section also displays the summary results that obtained and discussion is then made in which to explain the relationship between returns of nominal change in exchange rate per month and rubber price for Malaysia, Thailand, Philippines and Indonesia by using Bayesian method. The summary of parameter estimate for the selected countries that listed in table 2 is the results that obtained from posterior distribution table.

Table 2: Summary parameter estimate

\begin{tabular}{|c|c|c|c|c|c|}
\hline Component & Effect & $\begin{array}{l}\text { Estimate } \\
\text { Malaysia }\end{array}$ & Thailand & Philippines & Indonesia \\
\hline 1 & Intercept & -0.00187 & -0.00316 & -0.00314 & 0.000412 \\
\hline 1 & Rubber & -0.0525 & -0.0210 & -0.0256 & -0.0828 \\
\hline 2 & Intercept & 0.00323 & 0.0363 & 0.00934 & 0.00324 \\
\hline 2 & Rubber & 0.1049 & 0.1215 & 0.1082 & 0.1048 \\
\hline 1 & Variance & 0.0235 & 0.0236 & 0.0235 & 0.0240 \\
\hline 2 & Variance & 1.0455 & 1.0100 & 1.0470 & 1.0455 \\
\hline \multicolumn{2}{|c|}{ Mixing probability } & 0.9885 & 0.9885 & 0.9885 & 0.9885 \\
\hline
\end{tabular}

By referring to table 2, it can be concluded that both the intercept and rubber for component 1 at Malaysia, Thailand and Philippines are negative value. While these two effects are then displays positive value for component 2 . This indicates that the fluctuation of rubber price influence the exchange rate. Hence, it is reasonable because rubber is one of the top ten export products for Malaysia, Thailand and Philippines. While for Indonesia, the intercept in component 1 is positive value meanwhile the rubber displays negative value in component 1 . Then, both the intercept and rubber show positive value in component 2 and this illustrate that the flowing of rubber price may influence the exchange rate. In addition, the variance for both component 1 and component 2 at Malaysia, Thailand, Philippines and Indonesia are small. This indicates that the result of finding is valid, reliable and significant. The mixing probability that displays at table 2 denotes the weight for component 1 meanwhile the weight for component 2 is 0.0115 because the total weight for component is equal to one. This illustrated that the component 1 for all four countries are more dominant in compared to component 2 and this can be proved by referring to the mixing probability that listed in summary parameter estimate table above. Moreover, the mixing probability Markov Chain diagnostics plots for each country are displays at figure 1. In each figure, there are three plots which are trace, autocorrelation and density plot. The trace plot is placed at top of the Markov Chain diagnostics plot meanwhile the autocorrelation plot displayed on the lower left and the density plot placed on the lower right of the diagnostic plot. 
Figure 1: Markov Chain Diagnostic plot

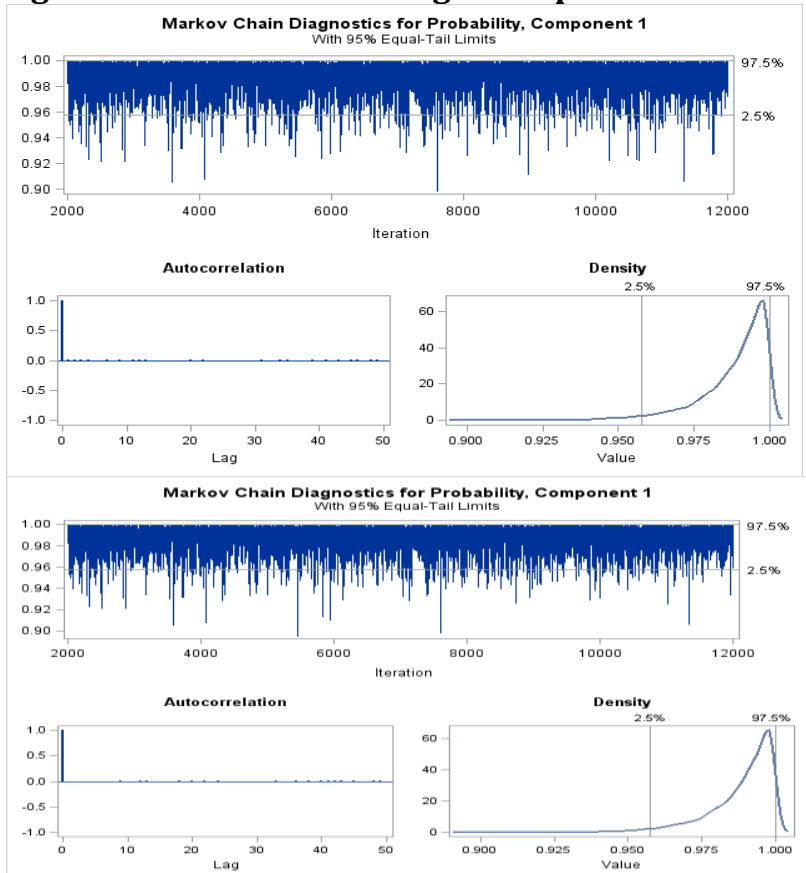

Malaysia

Thailand

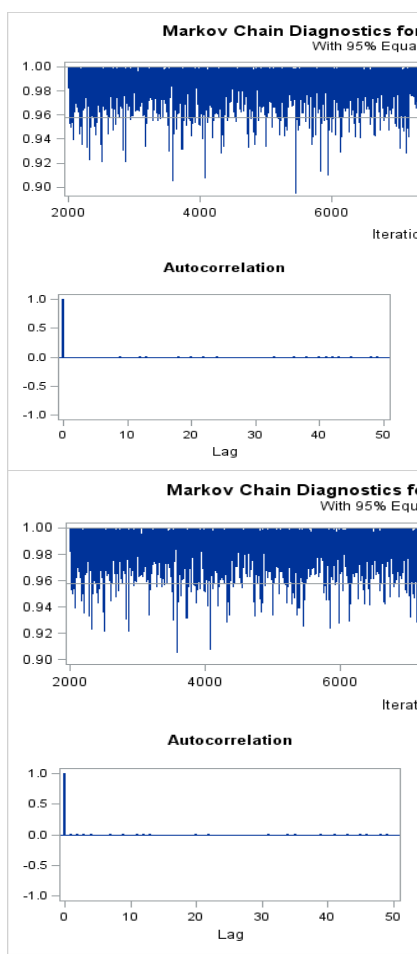

Philippines

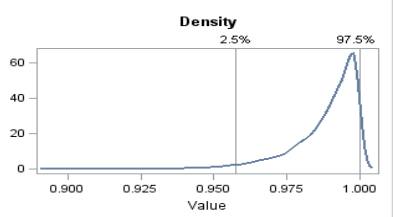

The diagnostics plots for all countries in figure 1 show constant alternation for the trace plot, a smooth unimodel distribution in the density plot and a quick drop-off in the autocorrelation plot. This indicates that the chain is successfully converged and the rubber price affects the exchange rate on the selected countries.

\section{Conclusion and Recommendations}

Bayesian method is applied in fitting finite mixture model in order to investigate the relationship between nominal changes in exchange rate per month and rubber price for four selected countries. The result 
depicts that the fluctuation of rubber price for all selected countries, includes Malaysia, Thailand, Philippines and Indonesia are effect on the exchange rate. This is reasonable because rubber is one of the top ten export products for all selected countries. Furthermore, Thailand is known as the world's largest rubber producer and exporter and then followed by Indonesia and Malaysia. Together, these three countries account more than $70 \%$ of world's rubber production and exportation. While Philippines also known as one of the top ten rubber producers in world. Thus, application of Bayesian method to real financial data produces more stable estimates that asymptotically efficient. Moreover, the further study can be carry out in studying other financial data or rubber price effect on stock market index. While the statistical method such as maximum likelihood estimation and L-moment method can be use instead of Bayesian method in studying the relationship between rubber price and exchange rate.

\section{References}

Abraham, B. \& Box, G. E. P. (1979). Bayesian analysis of some outlier problems in time series. Biometrika, $66,229-236$.

Akaike, H. (1974). A new look at statistical model identification. IEEE Transactions on Automatic Control, $19,716-722$.

Armero, C., Donato, G. G. \& Quilez, A. L. (2010). Bayesian methods in Cost-Effectiveness studies: Objectivity, Computation and other relevant aspects. Health Economics, 19, 629-643.

Berry, D. A. (2004). Bayesian Statistics and the Efficiency and Ethics of Clinical Trials. Statistical Science, 19(1), 175-187.

Cano, R., Sordo, C. \& Gutierrez, J. M. (2004). Applications of Bayesian Networks in Meteorology. In J. A. Gámez, et al. (Eds.) Advances in Bayesian Networks (pp. 309-327). Springer-Verlag.

Cipriani, M., Costantini, R. \& Guarino, A. (2012). A Bayesian approach to experimental analysis: trading in a laboratory financial market. Review of Economic Design, 16(2), 175-191.

Cooper, G. F. \& Herskovits, E. (1992). A Bayesian Method for the Induction of Probabilistic Networks from Data. Journal of Machine Learning, 9(4), 309-347.

Doss, H. \& Huffer, F. (2003). Monte Carlo Methods for Bayesian Analysis of Survival Data Using Mixtures of Dirichlet Process Priors. Journal of Computational and Graphical Statistics, 12(2), 282-307.

Feng, X. X. \& Xie, D. J. (2012). Bayesian Estimation of CIR Model. Journal of Data Science, 10, 271-280.

Jacquier, E. \& Polson, N. G. (2012). Asset Allocation in Finance: A Bayesian Perspective. In D. Dellaportas, N. Polson \& G. Stephen (Eds.). Hierarchinal models and MCMC: a Tribute to Adrian Smith. Oxford: Oxford University Press.

Jones, C. S. (1998). Bayesian Estimation of Continuous-Time Finance Models. Retrieved from http://wwwbcf.usc.edu/ christoj/pdf/hfa7.pdf

Kottas, A. (2006). Nonparametric Bayesian survival analysis using mixtures of Weibull distributions. Journal of Statistical Planning and Inference, 136(3), 578-596.

Laplace, P. S. (1986). Memoir on the Probability of the Causes of Events. Statistical Science, 1(3), 364-378.

Lindsay, B. G. (1989). Moment matrices: Applications in mixtures. Annual Statistics, 17, 722-740.

Liu, L. M. \& Tiao, G. C. (1980). Random coefficient first-order autoregressive models. Journal of Econometrics, 13, 305-325.

Madigan, D., Ryan, P., Simpson, S. E. \& Zorych, I. (2010). Bayesian methods in pharmacovigilance. In J. M. Bernardo, M. J. Bayarri, J. O. Berger, A. P. Dawid, D. Heckerman, A. F. M. Smith \& M. West (Eds.), Bayesian Statistics 9 (pp. 421-438). Oxford: Oxford University Press.

Martina, M. L. V., Todini, E. \& Libralon, A. (2008). Rainfall Thresholds for Flood Warning Systems: A Bayesian Decision Approach. Water Science and Technology Library, 63(3), 203-227.

McLachlan, G. J. \& Peel, D. (2000). Finite Mixture Models, New York: John Wiley \& Sons.

Schervish, M. J. \& Tsay, R. S. (1988). Bayesian Modeling and Forecasting in Autoregressive Models. In J. C. Spall, (Ed.), Bayesian Analysis of Time Series and Dynamic Models (pp. 23-52). New York: Marcel Dekker.

Schwarz, G. E. (1978). Estimating the dimension of a model. Annals of Statistics, 6(2), 461-464. 